\title{
Modification of the $B$ meson mass in a magnetic field from QCD sum rules
}

\author{
C. S. Machado ${ }^{*}$ and R. D. Matheus ${ }^{\dagger}$ \\ Instituto de Física Teórica, Universidade Estadual Paulista, R. Dr. Bento Teobaldo Ferraz, 271 - Bloco II, \\ Barra-Funda, São Paulo CEP 01140-070, Brazil \\ S. I. Finazzo ${ }^{\ddagger}$ and J. Noronha ${ }^{\S}$ \\ Instituto de Física, Universidade de São Paulo, R. do Matão Travessa R Nr.187, Cidade Universitária, \\ São Paulo CEP 05508-090, Brazil
}

(Received 18 July 2013; published 10 April 2014)

\begin{abstract}
In this paper, we extend the well-known QCD sum rules used in the calculation of the mass of heavy mesons to estimate the modification of the charged $B$-meson mass, $m_{B}$, in the presence of an external Abelian magnetic field, $e B$. Two simplifying limits were considered: the weak field limit, in which the external field satisfies $e B \ll m^{2}$ (with $m$ being any of the masses involved); and the strong field limit, in which the field strength is small in comparison to the bottom quark mass (or the $B$-meson mass) squared, but it is large compared to the mass of the light quarks, i.e., $m_{u, d}^{2} \ll e B \ll m_{b, B}^{2}$. We found that $m_{B}$ decreases with the magnetic field in both of these limits.
\end{abstract}

DOI: $10.1103 /$ PhysRevD.89.074027

PACS numbers: 12.38.-t, 12.38.Lg, 14.40.Nd

\section{INTRODUCTION}

Strong magnetic fields can be relevant to a number of physical systems. Some dense stars, such as highly magnetized neutron stars known as magnetars, can display magnetic fields as large as $e B \sim 1 \mathrm{MeV}^{2}[1] .{ }^{1}$ Of a more direct interest to particle physics are the electromagnetic fields produced in ultrarelativistic heavy ion collisions performed at the Large Hadron Collider (LHC) or at the Relativistic Heavy Ion Collider (RHIC). Noncentral collisions in these colliders can produce short-lived electromagnetic fields where the intensity of the magnetic field can be as large as $e B \sim m_{\pi}^{2} \sim$ $0.02 \mathrm{GeV}^{2}$ (at RHIC) or $e B \sim 15 m_{\pi}^{2} \sim 0.3 \mathrm{GeV}^{2}$ (at LHC) [2,3], where $m_{\pi}$ is the pion mass.

These field strengths are comparable with the hadronic mass scale and could have important phenomenological implications to the physics of hadrons in quantum chromodynamics (QCD). Much effort has been given towards understanding the effects of strong magnetic fields on the different phases of the QCD diagram [4], and recently, lattice QCD simulations with physical quark masses have determined how the deconfinement and chiral phase transitions are affected by strong magnetic fields [5].

The mass spectrum of the hadronic states, however, is set by the nonperturbative regime of QCD, and one has to look for the appropriate nonperturbative tools to describe it and include the effects of the magnetic field therein. For instance,

\footnotetext{
*camilasm@ift.unesp.br

†matheus@ift.unesp.br

stefanofinazzo@gmail.com

\$noronha@if.usp.br

${ }^{1}$ The field strength in the CGS system is $(e B)^{2} \sim 59.14 \times$ $10^{-22}(B / 1 \mathrm{G}) \mathrm{GeV}^{2}$; i.e, a magnetic field of magnitude $1.69 \times$ $10^{20} \mathrm{G}$ corresponds to $1 \mathrm{GeV}^{2}$.
}

one would expect that magnetic fields with values defined at the hadronic scale would modify the binding energy of the various hadronic states, which could then affect their masses.

Various methods have been successful in dealing with the hadronic spectrum over the years, such as, for instance, quark potential models [6], lattice QCD [7,8], and the QCD spectral sum rules (QCDSR) $[9,10]$. Weak external fields in the QCDSR were introduced in the past in order to analyze the magnetic moments of hadrons $[11,12]$. In this work, we propose a novel way to include an external magnetic field in the QCDSR formalism for the two-point correlators in order to study its effect on the masses of pseudoscalar $B^{ \pm}$mesons. We start in Sec. II with a short review on the QCDSR in the vacuum (i.e., in the absence of external fields, $e B=0$ ). The external field contributions are considered in Sec. III, and they enter the QCDSR in two ways.

First, the effects of an external magnetic field on the quark propagators are taken into account using the nonperturbative Schwinger propagator [13]. This modification describes how the perturbative sector of QCD changes due to the magnetic field. The complete calculation using the proper-time propagator is technically difficult, and we discuss a series of approximations that allow us to extract results in some limiting situations. The second modification introduced in this paper with respect to the usual QCDSR approach is the dependence of the condensates that parametrize the nonperturbative character of QCD with the magnetic field. The effect of magnetic fields on the chiral condensate was evaluated by a number of means, including chiral perturbation theory [14-16], Nambu-Jona-Lasinio models [17,18], and lattice QCD [19-24]. Using condensates that are functions of the field strength, we take into account the effects of the magnetic field on the longdistance, nonperturbative interactions of QCD. 
The numerical analysis of the QCDSR and the results for the masses are shown in Sec. IV, where we show that the magnetic field has the effect of lowering the masses of the heavy mesons studied, which is in agreement with recent potential model calculations $[25,26]$.

\section{QCD SUM RULES IN THE VACUUM}

QCDSR $[9,10]$ are based on the evaluation of the twopoint correlation function in the vacuum

$$
\Pi(q)=i \int d^{4} x e^{i q \cdot x}\left\langle 0\left|T\left\{j(x) j^{\dagger}(0)\right\}\right| 0\right\rangle,
$$

where $j(x)$ is an interpolating current carrying the quantum numbers of the hadron in question. For the $B^{ \pm}$mesons, we will use

$$
j(x)=\bar{q}_{a}(x) i \gamma_{5} Q_{a}(x),
$$

where $q$ is the light quark field, $Q$ is the heavy quark field, and $a$ is a color index. With this current in Eq. (1), the correlator can be written as

$$
\Pi(q)=\frac{i}{(2 \pi)^{4}} \int d^{4} k \operatorname{Tr}\left[S_{a b}^{q}(k) \gamma_{5} S_{b a}^{Q}(k+q) \gamma_{5}\right],
$$

where $S^{q}\left(S^{Q}\right)$ is the full propagator (considering all perturbative and nonperturbative effects and also, in the next sections, the interaction of the quark with the external magnetic field) for a light (heavy) quark.

Based on the principle of quark-hadron duality, which states that correlation functions of colorless currents in QCD can be described either in terms of quarks and gluons or in terms of hadronic degrees of freedom, the correlator [Eq. (1)] will be evaluated here in two different ways. On the one hand, we start from a perturbative description based on the quark and gluon degrees of freedom using Wilson's operator product expansion (OPE) [27] to evaluate Eq. (1) in the presence of nonzero vacuum condensates that act as a source for nonperturbative effects. The resulting expression for the correlation function is called the OPE side. On the other hand, on the phenomenological side, we use a description based on hadronic degrees of freedom by inserting a complete set of hadronic states in Eq. (1) to obtain the correlator in terms of a dispersion relation:

$$
\Pi^{\text {phen }}(k)=\int_{0}^{\infty} d s \frac{1}{s-k^{2}-i \epsilon} \rho(s),
$$

where $\rho(s)$ is the spectral density. The following parametrization is generally used for the spectral density:

$$
\rho^{\text {phen }}(s)=\frac{m_{H}^{4}}{m_{Q}^{2}} f_{H}^{2} \delta\left(s-m_{H}^{2}\right)+\theta\left(s-s_{0}\right) \rho^{\text {cont }}(s),
$$

where $m_{H}$ is the mass of the ground state of the hadron, $s_{0}$ is the continuum threshold, and $f_{H}$ is the coupling of this state with the current, which for heavy-light mesons is defined by $\langle 0|j| H\rangle=\frac{m_{H}^{2}}{m_{Q}+m_{q}} f_{H} \approx \frac{m_{H}^{2}}{m_{Q}} f_{H}$, and $m_{Q}$ and $m_{q}$ are the masses of the heavy and light quarks, respectively. This parametrization separates the contribution of the lowest-lying pole from that of the excited states, collectively called "the continuum." The parameter $s_{0}$ indicates when the excited states start to contribute significantly to the spectral density.

Then, the phenomenological side takes the form

$$
\Pi^{\text {phen }}(k)=\frac{m_{H}^{4} f_{H}^{2}}{m_{Q}^{2}\left(m_{H}^{2}-k^{2}\right)}+\int_{s_{0}}^{\infty} d s \frac{\rho^{\mathrm{cont}}(s)}{s-k^{2}} .
$$

The OPE side can also be written in terms of a dispersion relation:

$$
\Pi^{\mathrm{OPE}}(k)=\int_{s_{\min }}^{\infty} d s \frac{\rho^{\mathrm{OPE}}(s)}{s-k^{2}},
$$

where $\rho^{\mathrm{OPE}} \equiv \frac{\mathrm{Im} \Pi^{\mathrm{OPE}}}{\pi}$ and $s_{\min }=\left(m_{q}+m_{Q}\right)^{2}$. The principle of duality allows the identification $\rho^{\mathrm{OPE}}=\rho^{\text {phen }}$, which implies

$$
\int_{s_{0}}^{\infty} d s \frac{\rho^{\mathrm{OPE}}(s)}{s-k^{2}}=\int_{s_{0}}^{\infty} d s \frac{\rho^{\mathrm{cont}}(s)}{s-k^{2}}
$$

allowing us to cancel these terms on both sides of the equation:

$$
\Pi^{\mathrm{OPE}}(k)=\Pi^{\text {phen }}(k) .
$$

Taking the Borel of Eq. (9), we arrive at the sum rule

$$
\frac{m_{H}^{4}}{m_{Q}^{2}} f_{H}^{2} e^{-m_{H}^{2} / \bar{M}^{2}}=\int_{s_{\min }}^{s_{0}} d s \rho^{\mathrm{OPE}}(s) e^{-s / M^{2}},
$$

where $\bar{M}$ is the Borel mass $[9,10]$. Taking the derivative of Eq. (10) with respect to $1 / \bar{M}^{2}$ and dividing the resulting expression by Eq. (10), we get an explicit expression for the hadron mass:

$$
m_{H}^{2}=\frac{\int_{s_{\min }}^{s_{0}} d s \rho^{\mathrm{OPE}}(s) s e^{-s / \bar{M}^{2}}}{\int_{s_{\min }}^{s_{0}} d s \rho^{\mathrm{OPE}}(s) e^{-s / \bar{M}^{2}}} .
$$

In previous works [28-30], QCDSR results for heavylight states such as the $B$ meson have considered the effects of dimension- 4 condensates and radiative corrections. The inclusion of higher-dimensional condensates improves the stability of the QCDSR and, consequently, leads to more precise values for the mass. However, we have verified (see Sec. IVA) that the $B$-meson mass obtained with the OPE calculated up to dimension 3 is compatible with both the experimental result and previous QCDSR results, suffering 
only from increased dependence on the Borel mass. Since our aim is to establish a method for the inclusion of the strong magnetic fields in the QCDSR and get the first estimate of the change in the mass, the additional contributions are not included. The effect of the magnetic field on the dimension- 4 gluon condensate has been analyzed in Ref. [15] so that, in principle, one could include it in the same way done here in the case of the chiral condensate. However, the breaking of rotational symmetry will introduce new dimension- 4 condensates, ${ }^{2}$ previously forbidden by Lorentz invariance in the vacuum $(e B=0)$, and there is no information on the behavior of these condensates under strong magnetic fields. Fully introducing $g^{2}$ corrections (which include the gluon condensates and radiative corrections) would bring new unknown parameters into these calculations. With that in mind, in this first study we will consider the OPE expansion up to operators of dimension 3 , which is enough to study the overall change in mass when the magnetic field is introduced. In the vacuum, this means that one needs to consider only the identity operator and the quark condensate $\langle\bar{q} q\rangle$ in the calculations. We leave more precise predictions of mass values at specific field values for future work, when more data on the behavior of dimension- 4 condensates are available.

\section{QCD SUM RULES WITH MAGNETIC FIELDS}

In order to determine the contributions from the OPE to the correlation function in the presence of an external magnetic field, we will consider, as the "free" quark propagator, the full nonperturbative propagator computed by Schwinger [13] that describes the interaction of a spin$1 / 2$ field with the magnetic field. However, this propagator does not include the QCD interactions experienced by the quarks, which are parametrized here using the nonperturbative QCD condensates. The interactions of the magnetic field with the QCD vacuum are taken into account by considering the dependence of the condensates with the external field. On the phenomenological side, we will take the pole contribution as being given by the full propagator of a charged pseudoscalar meson in an external magnetic field.

\section{A. Quark propagator in the presence of an external magnetic field}

The Schwinger proper-time representation [13] describes the Feynman propagator of a spin-1/2 fermion with charge $e$ and mass $m$ in an external, constant, and uniform Abelian magnetic field. Considering the magnetic field in the $z$ direction and the symmetric gauge, i.e.,

\footnotetext{
${ }^{2}$ See Appendix A for an example of the same effect on dimension-3 condensates.
}

$\mathbf{A}=(-B y / 2, B x / 2,0)$, the Schwinger propagator can be written as ${ }^{3}$

$$
\begin{aligned}
S_{a b}(k)= & \delta_{a b} \int_{0}^{\infty} d s \exp \left[i s\left(k_{0}^{2}-k_{3}^{2}-k_{\perp}^{2} \frac{\tan (e B s)}{e B s}-m^{2}\right)\right] \\
& \times\left[\left(k^{0} \gamma^{0}-k^{3} \gamma^{3}+m\right)\left(1+\gamma^{1} \gamma^{2} \tan (e B s)\right)\right. \\
& \left.-k_{\perp} \cdot \gamma_{\perp}\left(1+\tan ^{2}(e B s)\right)\right] .
\end{aligned}
$$

We can also write the propagator as a sum over Landau levels [18]:

$$
S_{a b}(k)=i \delta_{a b} e^{-k_{\perp}^{2} /|e B|} \sum_{n=0}^{\infty}(-1)^{n} \frac{D_{n}(e B, k)}{k_{\|}^{2}-m^{2}-2|e B| n+i \epsilon},
$$

with

$$
\begin{aligned}
D_{n}(e B, k)= & \left(k^{0} \gamma^{0}-k^{3} \gamma^{3}+m\right) \\
& \times\left[\left(1-\gamma^{1} \gamma^{2} \operatorname{sign}(e B)\right) L_{n}\left(\frac{2 k_{\perp}^{2}}{|e B|}\right)\right. \\
& \left.-\left(1+i \gamma_{1} \gamma_{2} \operatorname{sign}(e B)\right) L_{n-1}\left(\frac{2 k_{\perp}^{2}}{|e B|}\right)\right] \\
& +4\left(k^{1} \gamma^{1}+k^{2} \gamma^{2}\right) L_{n-1}^{1}\left(\frac{2 k_{\perp}^{2}}{|e B|}\right),
\end{aligned}
$$

where $L_{n}^{a}$ are the associated Laguerre polynomials and $L_{n} \equiv L_{n}^{0.4}$ The form of the propagator in Eq. (12) is more convenient when considering weak fields $\left(e B \ll m^{2}\right)$, as it can be easily expanded in powers of $e B / m^{2}$. The alternative form in Eq. (14) is convenient when one is interested in a strong field limit $\left(e B \gg m^{2}\right)$, since in this case the lowest Landau level (LLL), given by $n=0$, dominates. We shall develop later in this section how these approximations are relevant to the study of the heavy mesons considered in this paper.

\section{B. Nonperturbative QCD contributions}

The Schwinger propagator in Eq. (12) takes into account in a nonperturbative manner all the effects coming from the external magnetic field on the quark propagators, but we have not taken into account the intrinsic nonperturbative QCD effects (and their

\footnotetext{
${ }^{3}$ We use a mostly minus signature for the Minkowski metric, and the four-vectors $v^{\mu} \equiv\left(v_{0}, v_{1}, v_{2}, v_{3}\right)$ are separated into parallel, $v_{\|} \equiv\left(v_{0}, 0,0, v_{3}\right)$, and perpendicular pieces, $v_{\perp} \equiv\left(0, v_{1}, v_{2}, 0\right)$, with respect to the direction of the magnetic field. The inner product is written as $u_{\mu} v^{\mu} \equiv u \cdot v=$ $u_{\|} \cdot v_{\|}-u_{\perp} \cdot v_{\perp}$, where $u_{\|} \cdot v_{\|} \equiv u_{0} v_{0}-u_{3} v_{3}$ and $u_{\perp} \cdot v_{\perp} \equiv$ $u_{1} v_{1}+u_{2} v_{2}$. Thus, for instance, $u_{\|}^{2}=u_{0}^{2}-u_{3}^{2}$ and $u_{\perp}^{2}=u_{1}^{2}+u_{2}^{2}$.

${ }^{4}$ For $n<0$, one defines $L_{n}=L_{n-1}^{1}=0$.
} 
modification due to the external field). In the QCDSR method, the nonperturbative aspects of QCD are accounted for by performing the OPE of the correlator and considering the vacuum expectation values of the local operators thus obtained.

Schematically, one can think of an expansion on the quark propagators themselves ${ }^{5}$ and write

$$
S_{a b, \alpha \beta}^{q}=S_{a b, \alpha \beta}^{q, \text { pert }}+\left\langle: q_{a \alpha}(x) \bar{q}_{b \beta}(0):\right\rangle,
$$

where $S_{a b, \alpha \beta}^{q, p e r t}$ is the perturbative propagator, which in the presence of a magnetic field corresponds to Eq. (12). As for the normal ordered term, up to condensates of dimension 3 , we get (see Appendix A for further details)

$$
\left\langle: q_{a \alpha}(x) \bar{q}_{b \beta}(0):\right\rangle \approx-\frac{\delta_{a b}}{12}\langle: \bar{q} q:\rangle \delta_{\alpha \beta}-\frac{\delta_{a b}}{12}\left\langle: \bar{q} \sigma_{12} q:\right\rangle \sigma_{\alpha \beta}^{12} .
$$

The nonperturbative QCD effects are parametrized by the condensates $\langle\bar{q} q\rangle$ and $\left\langle\bar{q} \sigma_{12} q\right\rangle$. The inclusion of the effects from the magnetic field on the condensate terms is done by taking $\langle\bar{q} q\rangle$ and $\left\langle\bar{q} \sigma_{12} q\right\rangle$ as functions of $e B$. The value used for the light quark chiral condensate in the absence of the magnetic field is $\langle\bar{q} q\rangle_{0}=$ $(-0.23)^{3} \mathrm{GeV}^{3}$ [31,32], while $\langle\bar{Q} Q\rangle \sim 0$ for heavy quarks [9,33]. For the ratio $\Sigma(e B) \equiv\langle\bar{q} q\rangle(e B) /\langle\bar{q} q\rangle_{0}$, we use two different parametrizations according to the intensity of the magnetic field. For magnetic fields such that $e B / m_{\pi}^{2} \ll 1$, we use the chiral perturbation theory result $[14,16]$

$$
\Sigma(e B)=1+\ln 2 \frac{e B}{16 \pi^{2} F_{\pi}^{2}} I_{H}\left(\frac{m_{\pi}^{2}}{e B}\right),
$$

where $F_{\pi} \sim 93 \mathrm{MeV}$ is the pion decay constant, $m_{\pi}=$ $140 \mathrm{MeV}$ is taken to be the pion mass, and

$$
I_{H}(y)=\frac{1}{\ln 2}\left[\ln (2 \pi)+y \ln \left(\frac{y}{2}\right)-y-2 \ln \Gamma\left(\frac{1+y}{2}\right)\right] .
$$

For magnetic fields $e B>1 \mathrm{GeV}^{2}$, we use a linear extrapolation of the lattice results in Ref. [19] (note, however, that the contribution from the condensates to the mass of $B$ mesons is very small, and thus, our final results for the masses are not sensitive to such an extrapolation). In the limit of weak fields, the lattice results are compatible with those found in chiral

\footnotetext{
${ }^{5}$ Note that the expansion is performed within the correlator, and one must be careful with OPE terms that potentially involve more than one propagator, such as the gluon condensate. However, since we are only considering condensates of dimension 3, such subtleties do not appear in our calculations.
}

perturbation theory [19]. Due to the Dirac matrix structure of the pseudoscalar current in Eq. (2), the term proportional to $\left\langle\bar{q} \sigma_{12} q\right\rangle$ results in a vanishing trace in Eq. (3), and thus, such a term does not enter into our calculations. However, for other types of interpolating currents [such as the vector mesons $\Upsilon(1 S)$ or $B^{*}$ ], the contribution from the $\left\langle\bar{q} \sigma_{12} q\right\rangle$ condensate may enter explicitly in the OPE and must be considered. With this possibility in mind, we remark that there are already lattice results for this quantity in the presence of a magnetic field [34].

The nonperturbative contribution to the correlator that comes from using the propagator in Eq. (15) in the OPE two-point function, after the Borel transform, is

$$
\hat{\Pi}_{\langle\overline{\mathrm{q}}\rangle}(\bar{M})=-m_{Q}\langle\bar{q} q\rangle e^{-m_{Q}^{2} / \bar{M}^{2}},
$$

where $m_{Q}$ is the heavy quark mass and $\bar{M}$ is the Borel mass.

\section{Weak field approximation}

In this paper, the weak field limit is defined by the condition $e B \ll m_{q}^{2} \ll m_{Q, B}^{2}$, where $m_{q}$ is the mass of the light quark, and $c$ is the charge of the light quark in units of the electron charge. This considerably simplifies the propagator in Eq. (12). We define $x \equiv s m^{2}$ (where $m$ here can be either one of the masses) and expand Eq. (12) in powers of $\left(\mathrm{ceB} / \mathrm{m}^{2}\right)$, up to quadratic order, obtaining $(e B>0)$

$$
\begin{aligned}
S_{a b}(k)= & \delta_{a b} \int_{0}^{\infty} d x \frac{e^{i x \alpha / m^{2}}}{m^{2}} \\
& \times\left[(k+m)+\left(k_{\|} \cdot \gamma_{\|}+m\right) \gamma^{1} \gamma^{2}\left(\frac{c e B x}{m^{2}}\right)\right. \\
& \left.-k_{\perp} \cdot \gamma_{\perp}\left(\frac{c e B x}{m^{2}}\right)^{2}\right],
\end{aligned}
$$

with

$$
\alpha \equiv k^{2}-m^{2}-\frac{k_{\perp}^{2}}{3}\left(\frac{c e B x}{m^{2}}\right)^{2}
$$

Evaluating the integrals, we obtain

$$
S(k)=S^{(e B)^{0}}(k)+S^{(e B)^{1}}(k)+S^{(e B)^{2}}(k),
$$

with

$$
S^{(e B)^{0}}(k)=i \frac{k+m}{k^{2}-m^{2}}
$$

and 


$$
\begin{aligned}
S^{(e B)^{1}}(k)= & \left(\frac{c e B}{m^{2}}\right)\left[-\left(k_{\|} \cdot \gamma_{\|}+m\right) \gamma^{1} \gamma^{2} \frac{m^{2}}{\left(k^{2}-m^{2}\right)^{2}}\right], \\
S^{(e B)^{2}}(k)= & \left(\frac{c e B}{m^{2}}\right)^{2}\left[-2 i k_{\perp}^{2}(-k+m) \frac{m^{4}}{\left(k^{2}-m^{2}\right)^{4}}\right. \\
& \left.+2 i k_{\perp} \cdot \gamma_{\perp} \frac{m^{4}}{\left(k^{2}-m^{2}\right)^{3}}\right] .
\end{aligned}
$$

For the perturbative part of the quark propagators, we use Eq. (22), which is a good approximation as long as $e B \ll m_{q}^{2} \ll m_{Q}^{2}$. These propagators are then inserted in the correlation function [Eq. (3)]. The momentum integrals are evaluated using a Feynman parametrization and a cutoff regularization - the details can be found in Appendix B. In the end, we arrive at

$$
\Pi^{\text {weak }}(q)=\Pi_{\text {pert }}^{(e B)^{0}}+\Pi_{1, \text { pert }}^{(e B)^{2}}+\Pi_{2, \text { pert }}^{(e B)^{2}}+\Pi_{\langle\bar{q} q\rangle},
$$

where

$$
\begin{aligned}
\Pi_{\mathrm{pert}}^{(e B)^{0}}= & \frac{3}{(2 \pi)^{2}} \int_{0}^{1} \mathrm{~d} x\left[2 \Delta-x(1-x) q^{2}-m_{q} m_{Q}\right] \log \Delta \\
\Pi_{1, \mathrm{pert}}^{(e B)^{2}} & =\frac{3}{(2 \pi)^{2}}(c C)(e B)^{2} \int_{0}^{1} \mathrm{~d} x(1-x) x \\
& \times\left[\frac{1}{\Delta}-\frac{\left(x^{2}-x\right) q_{\|}^{2}+m_{q} m_{Q}}{2 \Delta^{2}}\right]
\end{aligned}
$$

$$
\begin{aligned}
\Pi_{2, \text { pert }}^{(e B)^{2}}= & \frac{3 \cdot 2}{(2 \pi)^{2}}\left(c^{2}+C^{2}\right)(e B)^{2} \int_{0}^{1} \mathrm{~d} x\left\{\frac { x ^ { 3 } } { 3 } \left[\frac{3}{2 \Delta}+\frac{m_{q} m_{Q}+q_{\perp}^{2}\left(3-7 x+4 x^{2}\right)}{2 \Delta^{2}}\right.\right. \\
& \left.\left.-\frac{\left(x^{2}-x\right) q^{2}}{2 \Delta^{2}}+(1-x)^{2} q_{\perp}^{2} \frac{m_{q} m_{Q}-\left(x^{2}-x\right) q^{2}}{\Delta^{3}}+x^{2}\left[\frac{1}{\Delta}+\frac{\left(x^{2}-x\right) q_{\perp}^{2}}{\Delta^{2}}\right]\right]\right\},
\end{aligned}
$$

where $C$ is the charge of the heavy quark in units of the electric charge and

$$
\Delta \equiv\left(x^{2}-x\right) q^{2}+x m_{q}^{2}+(1-x) m_{Q}^{2}
$$

One can check that all linear terms in $e B$ have a vanishing Dirac trace. ${ }^{6}$ We have separated the order- $(e B)^{2}$ contribution in two terms for convenience. They come from different diagrams in Fig. 8 (in Appendix A) and are two distinct combinations on the fractional quark charges-one that depends on $(c C)\left(\Pi_{1, \text { pert }}^{(e B)^{2}}\right)$, and another that depends on $\left(c^{2}+C^{2}\right)\left(\Pi_{2, \text { pert }}^{(e B)^{2}}\right)$.

One may think that the expressions above could be easily simplified by taking the limit in which $m_{q} \rightarrow 0$. However, this approximation is not strictly allowed in the weak field limit, since in this case $e B \ll m_{q}^{2} \ll m_{Q}^{2}$. However, it is possible to rewrite the equations in terms of the dimensionless parameters $m_{q} / m_{Q}$ and $e B / m_{Q}^{2}$ to show that the terms proportional to $m_{q}$ only contribute to the real part of the correlator. Since we are only interested in $\rho^{\mathrm{OPE}}(q)$, which comes from the imaginary part of the correlator, we can safely take $m_{q} \rightarrow 0$ in this case. The result is an integral with a logarithmic term whose branch cut yields the

\footnotetext{
${ }^{6}$ The linear contribution in $e B$ comes from $\operatorname{Tr}\left[i \frac{(-k+m)}{k^{2}-m^{2}} \frac{(e B)}{m^{2}} \times\right.$ $\left.\left(-\left(k_{\|} \cdot \gamma_{\|}+m\right) \gamma^{1} \gamma^{2} \frac{m^{2}}{\left(k^{2}-m^{2}\right)^{2}}\right)\right]$. This is proportional either to the trace of an odd number of $\gamma^{\mu}$ matrices or to the off-diagonal elements of the metric tensor, so the contribution proportional to $e B$ vanishes.
}

imaginary part of the correlator plus polynomial terms without an imaginary part.

When $m_{q} \rightarrow 0$, the kinematic constraint in the $s=q^{2}$ integral is $s_{\min }=q_{\min }^{2}=m_{Q}^{2}$. We can use the relation $q^{2}=$ $q_{\|}^{2}-q_{\perp}^{2}$ to choose two of the three momenta as independent variables - it will be useful to choose $s=q^{2}$ and $q_{\perp}^{2}$. In the end, the spectral density of the OPE side is given by

$$
\begin{aligned}
\rho_{\text {pert }}^{\text {weak }}\left(s=q^{2}, q_{\perp}^{2}\right)= & \rho^{(e B)^{0}}\left(s, q_{\perp}^{2}\right)+\rho_{1}^{(e B)^{2}}\left(s, q_{\perp}^{2}\right) \\
& +\rho_{2}^{(e B)^{2}}\left(s, q_{\perp}^{2}\right),
\end{aligned}
$$

with

$$
\begin{gathered}
\rho^{(e B)^{0}}\left(s, q_{\perp}^{2}\right)=\frac{3}{8 \pi^{2}}\left[\frac{\left(s-m_{q}^{2}\right)^{2}}{s}+2 \frac{m_{Q} m_{q}}{s}\left(s-m_{Q}^{2}\right)\right] \\
\rho_{1}^{(e B)^{2}}\left(s, q_{\perp}^{2}\right)=-\frac{3}{4 \pi^{2}}(c C)(e B)^{2} \frac{m_{Q}^{2}}{s^{3}}\left(q_{\perp}^{2}\right)
\end{gathered}
$$

and

$$
\begin{aligned}
\rho_{2}^{(e B)^{2}}\left(s, q_{\perp}^{2}\right)= & -\frac{\left(c^{2}+C^{2}\right)(e B)^{2} m_{Q}^{4}}{4 \pi^{2}\left(m_{Q}^{2}-s\right)^{2}(s)^{3}} \\
& \times\left[6 m_{Q}^{4}-m_{Q}^{2}\left(s-14 q_{\perp}^{2}\right)-3 s\left(2 s+7 q_{\perp}^{2}\right)\right] .
\end{aligned}
$$

The Borel-transformed correlator in the weak field approximation is, at the one-loop level, 


$$
\hat{\Pi}_{\text {weak }}^{\text {OPE }}\left(\bar{M}, q_{\perp}^{2}\right)=\int_{s_{\text {min }}}^{s_{0}} d s\left[\rho_{\text {pert }}^{\text {weak }}\left(s, q_{\perp}^{2}\right) e^{-s / \bar{M}^{2}}\right]+\hat{\Pi}_{\langle\overline{\mathrm{q}} \mathrm{q}\rangle}(\bar{M}),
$$

where the continuum is already subtracted.

\section{Strong field approximation}

In the opposite limit, i.e., very strong magnetic fields such that $m_{q}^{2} \ll e B \ll m_{Q}^{2}$, we can use the alternative representation in Eq. (13) for the light quark propagator and keep only the lowest Landau level when $c e B / m_{q}^{2} \gg 1$ [18]. In other words, we truncate the Landau sum for the light quark propagator [Eq. (13)] at $n=0$ :

$S_{a b}^{(0)}(k)=i \delta_{a b} e^{-k_{\perp}^{2} /(c e B)}\left(\frac{k_{\|} \cdot \gamma_{\|}+m_{q}}{k_{\|}^{2}-m_{q}^{2}}\right)\left(1-i \gamma^{1} \gamma^{2}\right)$,

where $S_{a b}^{(0)}$ is the quark propagator truncated at the lowest Landau level. With respect to the heavy quark mass, the magnetic field is not strong, $e B \ll m_{Q}^{2}$, and we can still use the Taylor expansion in Eq. (22). With these propagators, we obtain

$$
\Pi^{\text {strong }}(q)=\bar{\Pi}_{\text {pert }}^{(e B)^{0}}+\bar{\Pi}_{1, \text { pert }}^{(e B)^{1}}+\bar{\Pi}_{2, \text { pert }}^{(e B)^{2}}+\Pi_{\langle\bar{q} q\rangle},
$$

where

$$
\bar{\Pi}_{\text {pert }}^{(e B)^{0}}(q)=3 \cdot 4 i \int \frac{d^{2} k_{\perp}}{(2 \pi)^{2}} e^{-\frac{k_{\perp}^{2}}{c e B}} \int \frac{d^{2} k_{\|}}{(2 \pi)^{2}} \frac{k_{\|}^{2}+k_{\|} \cdot q_{\|}}{\left((k+q)^{2}-m_{Q}^{2}\right) k_{\|}^{2}}
$$

and

$$
\begin{aligned}
\bar{\Pi}_{1, \text { pert }}^{(e B)^{1}}(q)= & -3 \cdot 4 i \frac{C e B}{m_{Q}^{2}} m_{Q}^{2} \int \frac{d^{2} k_{\perp}}{(2 \pi)^{2}} e^{-\frac{k_{\perp}^{2}}{c e B}} \\
& \times \int \frac{d^{2} k_{\|}}{(2 \pi)^{2}} \frac{k_{\|}^{2}+k_{\|} \cdot q_{\|}}{\left((k+q)^{2}-m_{Q}^{2}\right) k_{\|}^{2}},
\end{aligned}
$$

where we take $m_{q}=0$. One can show that the term proportional to $\left(e B / m_{Q}^{2}\right)^{2}, \bar{\Pi}_{2 \text {,pert }}^{(e B)^{2}}(q)$, vanishes after taking the Dirac trace. The $k_{\|}$integral can be done with the Feynman parametrization. The integral in the Feynman parameter results in a logarithmic term only for $\bar{\Pi}_{\text {pert }}^{(e B)^{0}}(q)$, from which we extract the imaginary part, and thus the OPE spectral density. The $\bar{\Pi}_{1, \text { pert }}^{(e B)^{1}}(q)$ integral is real and does not contribute to the imaginary part. Therefore, the spectral density of the OPE side in this strong field limit is given by

$$
\begin{aligned}
\rho_{\text {pert }}^{\text {strong }}\left(s=q^{2}, q_{\perp}\right)= & \frac{3}{2 \pi} e^{-q_{\perp}^{2} /(c e B)} \int_{0}^{\sqrt{s+q_{\perp}^{2}-m_{Q}^{2}}} d k_{\|} k_{\|} e^{-\frac{k_{\perp}^{2}}{c e B}} \\
& \times I_{0}\left(\frac{2 k_{\|} \sqrt{q_{\perp}^{2}+s}}{c e B}\right)
\end{aligned}
$$

where $I_{0}$ is the modified Bessel function. Finally, the Borel transformed correlator in the strong field approximation is, at the one-loop level,

$\hat{\Pi}_{\text {strong }}^{\mathrm{OPE}}\left(\bar{M}, q_{\perp}\right)=\int_{s_{\min }}^{s_{0}} d s\left[\rho_{\text {pert }}^{\text {strong }}\left(s, q_{\perp}\right) e^{-s / \bar{M}^{2}}\right]+\hat{\Pi}_{\langle\bar{q} q\rangle}(\bar{M})$,

where the continuum is already subtracted.

\section{E. The phenomenological side}

Since we are dealing with a charged (pseudo)scalar meson, we can use the Schwinger propagator for a spin-0 particle to describe the pole that appears in the phenomenological part of the QCDSR:

$$
\begin{aligned}
G(q)= & -i \int_{0}^{\infty} \frac{d s}{\cos (e B s)} \\
& \times \exp \left[-i s\left(m_{H}^{2}-q_{\|}^{2}+\frac{\tan (e B s)}{e B s} q_{\perp}^{2}\right)\right] ;
\end{aligned}
$$

here, $m_{H}$ is the mass of the hadronic state (in the present case, $m_{H}=m_{B}$ ).

Although this propagator is fully nonperturbative with respect to the external magnetic field, its full form is rather complicated to implement in the evaluation of the correlation function [Eq. (1)]. Since $m_{H}^{2} \gg e B$ in both scenarios explored on the OPE side, in this paper we expand the charged pseudoscalar propagator in powers of $e B / m_{H}^{2}$ in the phenomenological part of the QCDSR. Given that magnetic fields of the order $e B \sim m_{\pi}^{2}[2,3,35,36]$ are the most relevant for the study of heavy ion collisions at RHIC and LHC, in Ref. [37] the calculations presented in this paper will be generalized to consider effects from magnetic fields of arbitrary strength.

Therefore, we expand the propagator in Eq. (42) in powers of $e B / m_{H}^{2}$, and the final result up to order $(e B)^{2}$ is

$$
G(q)=\frac{1}{q^{2}-m_{H}^{2}}-(e B)^{2}\left[\frac{1}{\left(q^{2}-m_{H}^{2}\right)^{3}}+\frac{2 q_{\perp}^{2}}{\left(q^{2}-m_{H}^{2}\right)^{4}}\right]
$$

The Borel transform of the pole phenomenological side is then given by 
$\hat{\Pi}^{\text {phen }}\left(\bar{M}^{2}\right)=\frac{m_{H}^{4}}{m_{Q}^{2}} f_{H}^{2} e^{-m_{H}^{2} / \bar{M}}\left[1-(e B)^{2}\left(\frac{1}{2 \bar{M}^{4}}+\frac{q_{\perp}^{2}}{3 \bar{M}^{6}}\right)\right]$,

where the continuum is already subtracted.

\section{NUMERICAL RESULTS}

\section{A. Vacuum}

Since we are interested in the effect of the magnetic field on the meson mass, we will normalize our calculations by its vacuum $(e B=0)$ value, also determined via the QCDSR. Thus, we will briefly review the numerical results for the mass of the charged $B$ meson computed via the QCDSR in the absence of a magnetic field, which was already studied in Refs. [28-30].

We fixed $\langle\bar{q} q\rangle=(-0.23)^{3} \mathrm{GeV}$ and the quark masses $m_{b}=4.24 \mathrm{GeV}$ and $m_{u, d} \approx 0$. These values were chosen to mantain consistency with other QCDSR calculations [31,32]. The continuum threshold $s_{0}$ is a free parameter fixed using the phenomenological rule $\left(m_{H}+400\right) \mathrm{MeV} \lesssim$ $\sqrt{s_{0}} \lesssim\left(m_{H}+800\right) \mathrm{MeV}[31,38]$, which is an appropriate

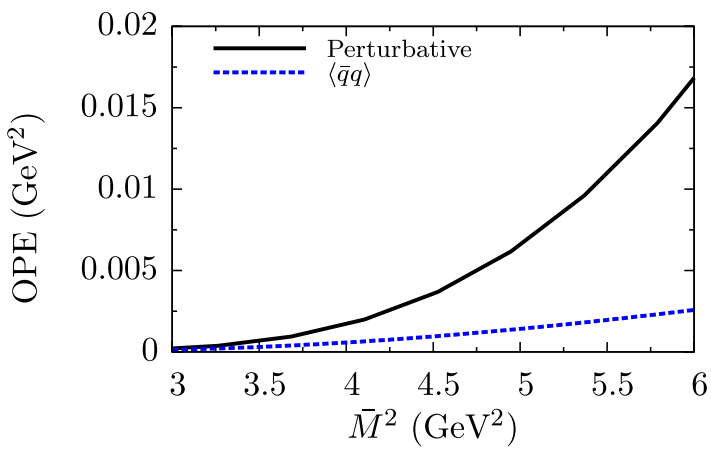

(a) way of fixing $s_{0}$ when the $s_{0}$-stable region falls outside the allowed $M^{2}$ window (explained below). This is the case for heavy-light mesons, as noted in Ref. [28].

In the QCDSR approach, there is an interplay between the convergence of the OPE (valid for large $-q^{2}$ or large Borel mass $\bar{M}^{2}$ ) and the contribution from the continuum of excited states (which is smaller than the pole contribution for low $-q^{2}$ or low $\bar{M}^{2}$ ). The OPE convergence is estimated by requiring that the contribution from the condensates of dimension 3 to the OPE be less than $33 \%$ of the perturbative contribution-this gives a lower limit to the Borel mass $\bar{M}_{\min }$ [see Fig. 1(a)]. An upper limit $\bar{M}_{\max }$ is determined by requiring that the contribution from the pole be larger than that from the continuum [see Fig. 1(b)]. We obtain $\bar{M}_{\min }^{2} \sim 4 \mathrm{GeV}^{2}$ [Fig. 1(a)] and $\bar{M}_{\max }^{2} \sim 8 \mathrm{GeV}^{2}$ for $\sqrt{s_{0}}=$ 6.0 GeV. The interval determined by $\bar{M}_{\min }$ and $\bar{M}_{\max }$ is called the Borel window, and the procedure explained above is used to fix it throughout this work.

With the Borel window fixed, we can determine the hadronic parameters (mass and coupling of the $B$ meson) by averaging the values in the Borel window [Fig. 2(a) for the mass and Fig. 2(b) for the coupling]. We obtain $m_{B}=5.25 \mathrm{GeV}$ and $f_{B}=0.29 \mathrm{GeV}$, which agree with

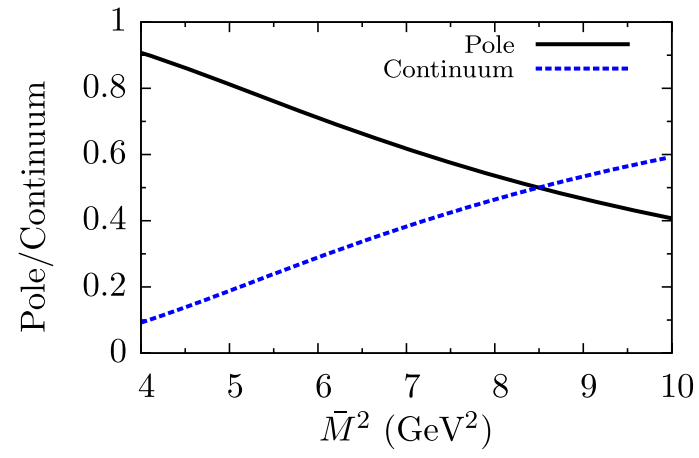

(b)

FIG. 1 (color online). (a) Convergence of the OPE expansion and (b) pole dominance in the absence of a magnetic field.

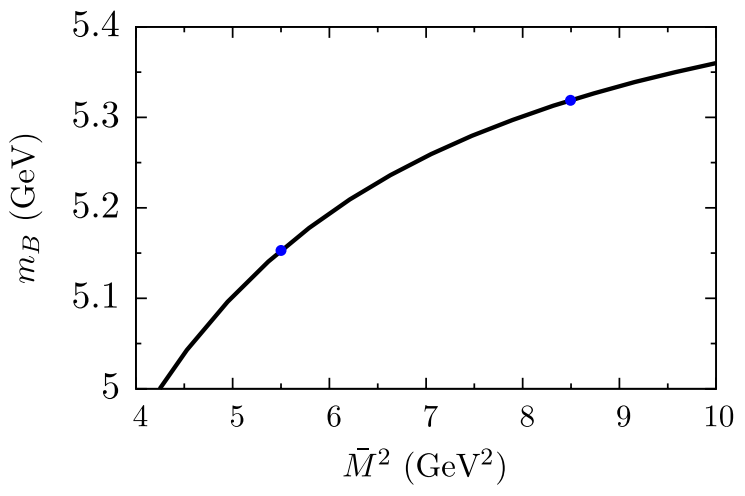

(a)

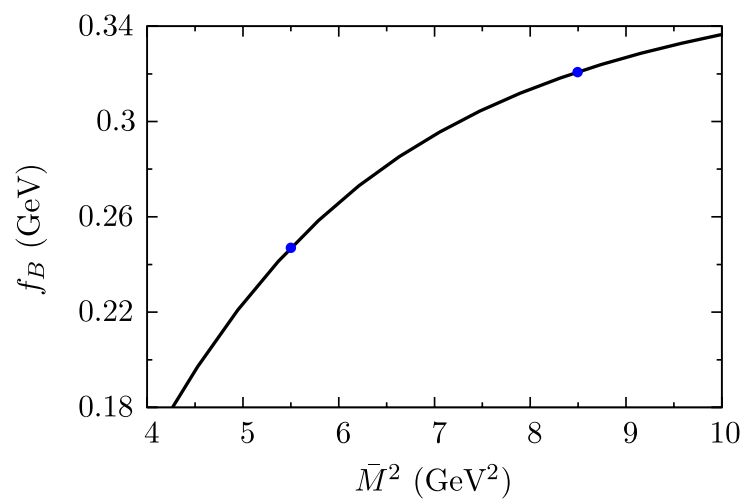

(b)

FIG. 2 (color online). Dependence of the mass (a) and coupling constant (b) of the $B$ meson, as functions of the Borel mass, in the absence of a magnetic field. The points indicate the Borel window. 


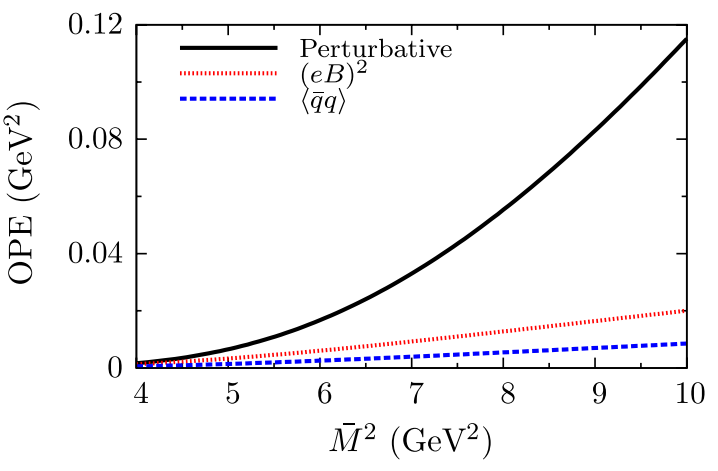

(a)

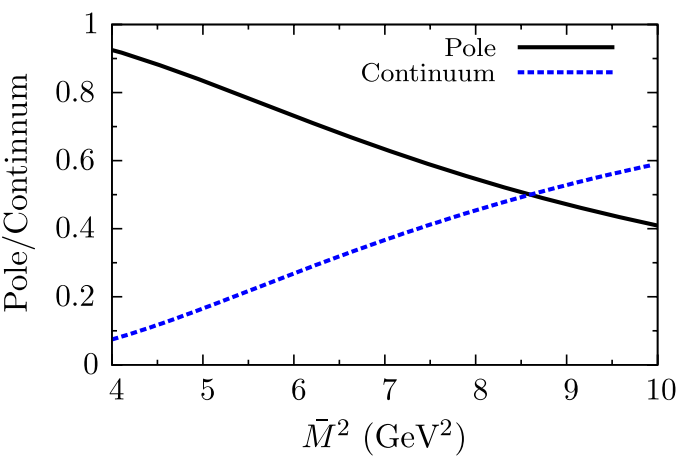

(b)

FIG. 3 (color online). (a) OPE convergence and (b) pole dominance for $e B=4 \times 10^{-8} \mathrm{GeV}^{2}$ and $q_{\perp}^{2}=1 \mathrm{GeV}^{2}$.

the experimental values [39] and the results in Refs. [28,29].

The errors in the QCDSR calculations come mainly from the truncation of the OPE expansion, from the choice of the continuum threshold $\left(s_{0}\right)$, and from the uncertainties in the values of the quark masses and condensates. These errors can be estimated by varying those parameters within their uncertainties. In this work, we will not make an estimate of QCDSR intrinsic errors, since we are interested in the ratios between sum-rules calculations with and without the effects of magnetic fields. The effect of those errors would be to create a narrow bundle of similar curves where now we have just one curve. As this would happen both in the vacuum and in the presence of the magnetic field, the central values for the ratios would not be affected.

\section{B. Weak magnetic fields}

The sum rules for the weak magnetic field case are obtained by equating Eqs. (35) and (44):

$$
\begin{aligned}
& \hat{\Pi}_{\langle\overline{\mathrm{q} q}\rangle}(\bar{M})+\int_{s_{\text {min }}}^{s_{0}} d s e^{-s / \bar{M}^{2}} \rho_{\text {pert }}^{\text {weak }}\left(s, q_{\perp}\right) \\
& \quad=\frac{m_{H}^{4}}{m_{Q}^{2}} f_{H}^{2} e^{-m_{H}^{2} / \bar{M}^{2}}\left[1-(e B)^{2}\left(\frac{1}{2 \bar{M}^{4}}+\frac{q_{\perp}^{2}}{3 \bar{M}^{6}}\right)\right],
\end{aligned}
$$

where $\hat{\Pi}_{\langle\overline{\mathrm{q}} \mathrm{q}\rangle}(\bar{M})$ is given by Eq. (19). In Eq. (45), the parameters we want to discover (for a given Borel mass $\bar{M}$ ) are the meson mass $m_{H}$ and the coupling constant $f_{H}$. As in the vacuum case, by differentiating Eq. (45) with respect to $1 / \bar{M}^{2}$, we can obtain a second equation to solve for $m_{H}$ and $f_{H}$. However, due to the more complex dependence of the phenomenological side on $\bar{M}$, we cannot eliminate $f_{H}$ from Eq. (45) by the same procedure done in the vacuum. Thus, we numerically solve Eq. (45) and its derivative with respect to $1 / \bar{M}^{2}$ to obtain simultaneously $m_{H}$ and $f_{H}$. As a consistency check, we verify that this procedure yields the same numerical results found for the vacuum in Sec. IV A.
For this initial study, we fix $e B=2 \times 10^{-6} m_{\pi}^{2} \sim$ $4 \times 10^{-8} \mathrm{GeV}^{2}$ and $q_{\perp}^{2}=1 \mathrm{GeV}^{2}$ - this last choice reflects a typical hadronic scale. The continuum threshold was chosen to be the same as that of the vacuum calculation, $s_{0}^{1 / 2}=(5.3 \pm 0.6) \mathrm{GeV}$. This approximation is justified, since the change in the mass due to the effect of the magnetic field, in the weak field limit, is expected to be small and thus not expected to greatly modify the threshold for contributions from the excited states. The convergence of the OPE is shown in Fig. 3(a), and the pole dominance can be seen in Fig. 3(b). The result for $m_{B}$ for these fixed values of $e B$ and $q_{\perp}^{2}$ is shown in Fig. 4, along with the respective Borel window. For magnetic fields larger than $\sim 4 \times 10^{-8} \mathrm{GeV}^{2}$, the contribution from the term $\sim(e B)^{2}$ is larger than the vacuum term, signaling the breakdown of our weak field expansion for the light quark propagator.

A more systematic study can be done to investigate the role of the choice of $q_{\perp}^{2}$ by fixing $e B \sim 2 \times 10^{-6} m_{\pi}^{2}$ and varying $q_{\perp}^{2}$. The results are shown in Fig. 5(a), where the computed masses and couplings are normalized by the vacuum results. One can see that $m_{B}$ is quite sensitive to the choice of $q_{\perp}^{2}$. Nevertheless, for any choice of $q_{\perp}^{2}$, the effect of the magnetic field is to lower $m_{B}$. This is consistent with the "Zeeman" splitting found for the $\rho$-meson mass in

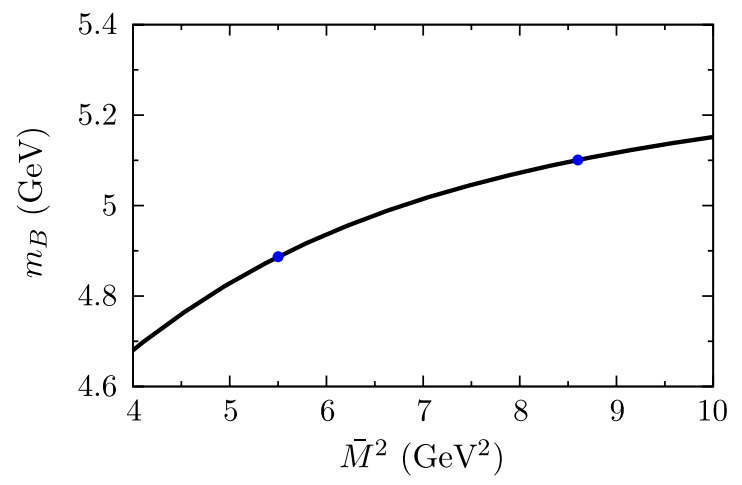

FIG. 4 (color online). Mass of the $B$ meson, $m_{B}$, as a function of the Borel mass $M^{2}$ for $e B=4 \times 10^{-8} \mathrm{GeV}^{2}$ (the weak field limit) and $q_{\perp}^{2}=1 \mathrm{GeV}^{2}$. The points indicate the Borel window. 


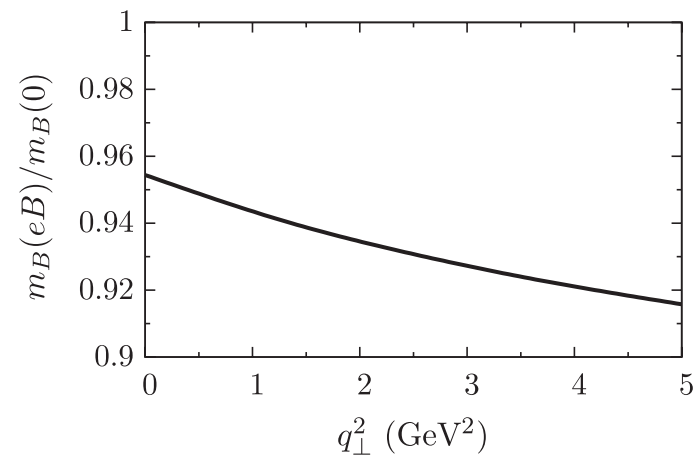

(a)

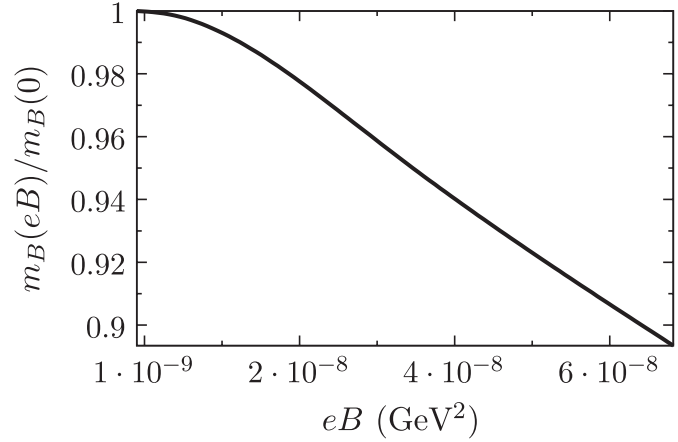

(b)

FIG. 5. (a) $B$-meson mass, $m_{B}$, as a function of the perpendicular momentum for a fixed magnetic field of $e B=2 \times 10^{-6} m_{\pi}^{2}$ (the weak field limit) and (b) dependence of $m_{B}$ on the magnetic field for a fixed perpendicular momentum $q_{\perp}^{2}=1 \mathrm{GeV}^{2}$.

the presence of magnetic fields $[25,40,41]$. However, in the QCDSR approach, we capture only the hadron ground state, and thus, one should expect to obtain only the lower meson mass.

With these observations in mind, we fix $q_{\perp}^{2}$ to be $1 \mathrm{GeV}^{2}$ and vary $e B$. The results are shown in Fig. 5(b). One can see that $m_{B}$ decreases with increasing $e B$, as expected.

\section{Strong magnetic fields}

In the strong magnetic field limit, the calculation is entirely analogous to the one realized in the preceding subsection, except that now we use Eq. (41) for the OPE side. In this case, we are within the limits $m_{q}^{2} \ll e B \ll m_{Q}^{2}$. By varying $e B$, we see that to have a valid Borel window, we have to limit $e B$ to be in the range $e B \sim 50 m_{\pi}^{2} \sim 1 \mathrm{GeV}^{2}$ and $e B \sim 200 m_{\pi}^{2} \sim 4 \mathrm{GeV}^{2}$. In Figs. 6(a) and 6(b), we show the convergence of the OPE and the dominance of the pole over the continuum for $e B=75 m_{\pi}^{2} \sim 1.5 \mathrm{GeV}^{2}$ and $q_{\perp}^{2}=0.5 \mathrm{GeV}^{2}$.

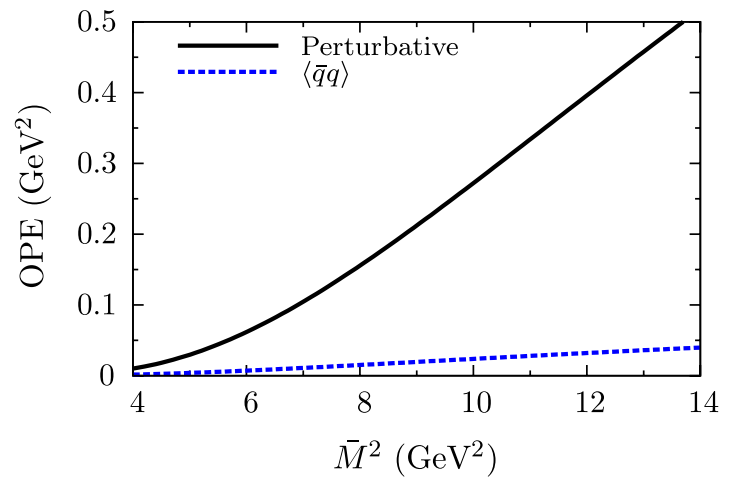

(a)
The continuum threshold, $s_{0}$, is one of the main sources of error in the QCDSR approach, and thus, one needs to be careful with the choice of this parameter in the strong field limit. Using the standard phenomenological estimate $\left(m_{H}+400\right) \mathrm{MeV} \lesssim \sqrt{s_{0}} \lesssim\left(m_{H}+800\right) \mathrm{MeV}$ as a guide, we chose, for $e B=1.0 \mathrm{GeV}^{2}, e B=2.5 \mathrm{GeV}^{2}$, and $e B=4.0 \mathrm{GeV}^{2}$, three values of $s_{0}$ which satisfy $\sqrt{s_{0}} \sim\left(m_{H}+600\right) \mathrm{MeV}$, using an interpolation of $s_{0}$ for intermediate values of $e B$. To analyze the sensitivity of the results with $s_{0}$, we also repeated this analysis for values of $\sqrt{s_{0}}$ in the range defined by $\sim\left(m_{H}+400\right) \mathrm{MeV}$ and $\sim\left(m_{H}+800\right) \mathrm{MeV}$, yielding one lower- and two upperlimit curves of continuum thresholds, $\sqrt{s_{0}}(e B), \sqrt{s_{0, \text { min }}}$ and $\sqrt{s_{0, \max }}$, respectively.

The results for the mass as a function of $e B$ and $q_{\perp}^{2}$ with the three values of $s_{0}$ are shown in Figs. 7(a) and 7(b). Note that in the strong field limit, $m_{B}$ displays the same qualitative behavior as a function of $e B$ as observed in the weak field case. However, in contrast with the weak field result [Fig. 5(b)], $m_{B}$ is found to be less sensitive to the choice of $q_{\perp}^{2}$.

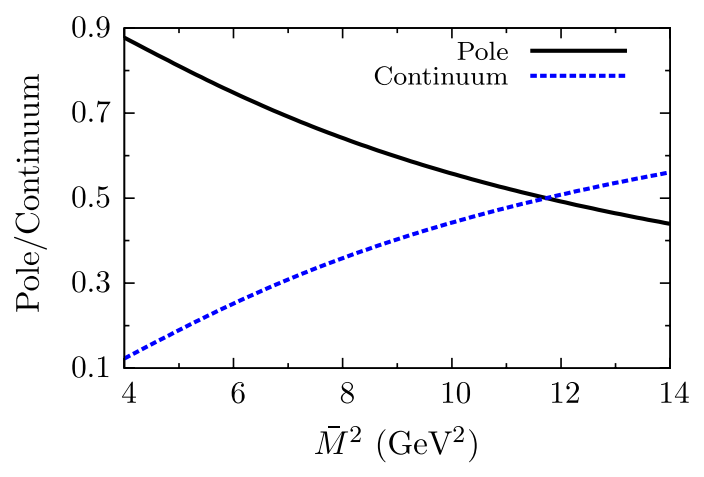

(b)

FIG. 6 (color online). (a) OPE convergence and (b) pole dominance for $e B=1.5 \mathrm{GeV}^{2}$ (strong field limit) and $q_{\perp}^{2}=0.5 \mathrm{GeV}^{2}$. 


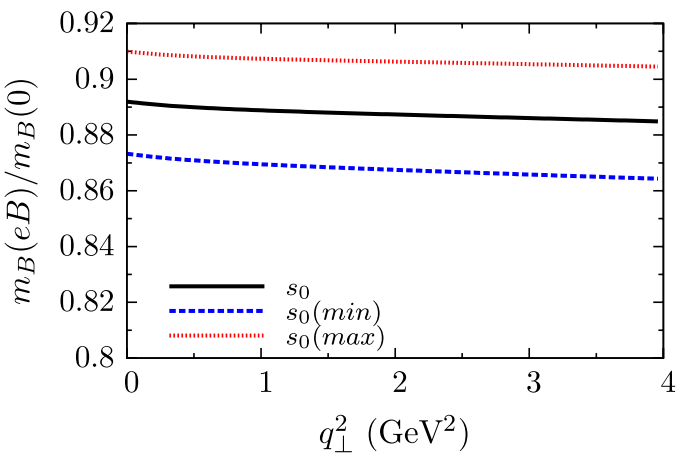

(a)

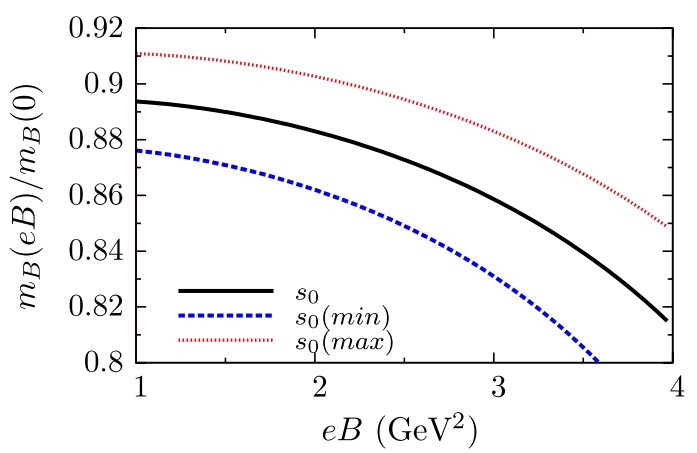

(b)

FIG. 7 (color online). (a) Mass of the $B$ meson, $m_{B}$, as a function of the perpendicular momentum $q_{\perp}^{2}$ for fixed $e B=1.5 \mathrm{GeV}^{2}$ (strong field limit), and (b) as a function of the magnetic field $e B$ for fixed $q_{\perp}^{2}=0.5 \mathrm{GeV}^{2}$. The curves correspond to the central $\sqrt{s_{0}} \sim\left(m_{H}+600\right) \mathrm{MeV}$, lower $\sqrt{s_{0}} \sim\left(m_{H}+400\right) \mathrm{MeV}$, and upper $\sqrt{s_{0}} \sim\left(m_{H}+800\right) \mathrm{MeV}$ interpolated continuum threshold $s_{0}(e B)$ curves, as described in the main text.

\section{CONCLUSIONS}

In this paper, we have introduced a modification of the QCDSR method in order to estimate the effects of external magnetic fields on the mass of charged $B$ mesons. The effect of such fields has been taken into account via two distinct modifications. First, the quark propagators (on the OPE side) and the meson propagator (on the phenomenological side) were modified using the proper-time representation introduced by Schwinger [13], which gives the exact propagators for fermions and scalar particles in the presence of a constant and uniform magnetic field. Secondly, the quark condensate, which encodes the nonperturbative aspect of QCD, has been replaced by its magnetic-field-dependent value, and the same approach could be used for higher-dimensional condensates. While these modifications include all the effects of a constant magnetic field in the QCDSR method, the full calculation using the complete proper-time propagators is technically difficult to implement. In this first study, we decided to restrict the calculation to some limiting situations in order to gain qualitative insight and order-of-magnitude estimates of the possible effects. Two such simplifying limits were considered: (1) the "weak field" limit, where the external field satisfies $e B \ll m^{2}$ (with $m$ being any of the masses involved, including both of the quarks and the meson), and (2) the "strong field" limit, where the field strength is still small compared to the bottom quark mass or the $B$-meson mass squared, but still large enough compared to the light quarks, i.e., $m_{u, d}^{2} \ll e B \ll M_{b, B}^{2}$.

In the "weak field" limit, we can expand all proper-time propagators in powers of $c e B / m^{2}$ (with $m$ being the mass of a given propagator), which greatly simplifies the calculation. We have kept terms up to $\left(e B / m^{2}\right)^{2}$ and evaluated the QCDSR with condensates up to dimension 3. Surprisingly enough, we have found sizable effects already with considerably weak fields $\left(e B \sim 2 \times 10^{-6} m_{\pi}^{2}\right)$. The effect of the field is to lower the meson mass. This result agrees with the expectation that the magnetic field splits the meson into two states, and the fact that the QCDSR only consider the lowest-lying state. The surprising feature found here is the magnitude of the mass suppression, which is about $10 \%$. We also found a strong dependence of the mass with respect to the meson momentum perpendicular to the magnetic field, which might have some phenomenological implications. Our calculations behave properly in the limit of $e B \rightarrow 0, q_{\perp} \rightarrow 0$, falling back to the usual QCDSR results.

In the "strong field" limit, we considered fields of the order $e B \sim 75 m_{\pi}^{2}$. In this limit, we can still expand the propagators of the heavy quark and the meson in the same way we did for the previous case. The light quark propagator, on the other hand, can be written as a sum over Landau levels, and for such a strong field, we assumed that only the lowest Landau level contributed significantly, which allowed us to truncate the sum to its first term. In this approximation, we found that the decrease in the $B$-meson mass is in the $10 \%$ to $20 \%$ range depending on the field strength, the perpendicular momentum, and the intrinsic QCDSR parameters. This is not a large effect, especially considering the results found in the weak field limit. It seems that most of the magnetic field effects take place at smaller field values, with the mass changing at a slower pace after that. That might indicate the presence of a saturation mechanism that stabilizes the meson mass as a function of the magnetic field, but a more complete calculation (valid for arbitrary values of the magnetic field) is needed to verify if that is indeed the case.

The values of magnetic fields relevant to ultrarelativistic heavy ion collisions, $e B \sim 1-15 m_{\pi}^{2}$, are in between the two sets of values considered in this paper. Although we do not expect the effects of the magnetic field to change qualitatively the result found here, a more complete calculation valid for arbitrary values of the magnetic field is needed to confirm this expectation. Such a calculation is also desirable, since it could be used to study other mesons that do 
not have the same separation of scales present in $B$ mesons (which justified our approximations). We are currently tackling the more general calculations that include the explicit sum over Landau levels, and we intend to present the results in a future publication [37].

\section{ACKNOWLEDGMENT}

This work has been partly supported by Fundação de Amparo à Pesquisa do Estado de São Paulo (FAPESP) and Conselho Nacional de Desenvolvimento Científico e Tecnológico $(\mathrm{CNPq})$. C. S. M. is supported by FAPESP under Contracts No. 2011/05619-3 and No. 2012/21627-9. S. I. F. is supported by FAPESP under Contract No. 2011/ 21691-6. The authors thank F. S. Navarra and I. A. Shovkovy for discussions about the QCD condensates in a magnetic field and M. Nielsen and R. Higa for comments on the manuscript.

\section{APPENDIX A: THE OPE FOR THE QUARK PROPAGATOR}

In this appendix, we work out the OPE expansion for the quark propagator in a constant, homogeneous magnetic field, including the effects of condensates of dimension 3. The procedure we follow can be extended to include condensates of higher dimension. However, note that in general, new condensates appear due to the magnetic field, and for dimensions higher than 4 , one does not yet have estimates for these new condensates in the presence of a magnetic field.

We start with the propagator for a quark field $q$ :

$$
\begin{aligned}
S_{a b, \alpha \beta}^{q} & \equiv\left\langle\Omega\left|T\left\{q_{a \alpha}(x) \bar{q}_{b \beta}(0)\right\}\right| \Omega\right\rangle \\
& =\left\langle 0\left|T\left\{q_{a \alpha}(x) \bar{q}_{b \beta}(0)\right\}\right| 0\right\rangle+\left\langle\Omega\left|: q_{a \alpha}(x) \bar{q}_{b \beta}(0):\right| \Omega\right\rangle,
\end{aligned}
$$

where $|\Omega\rangle$ is the true vacuum, $|0\rangle$ is the perturbative vacuum, $T$ is the time-ordering operator, $a, b=1,2,3$ are color indices, and $\alpha=1,2,3,4$ is a Dirac matrix index. The first term in the second line is the perturbative propagator. In our case, instead of the free propagator, we will use the Schwinger proper-time propagator for a fermion in a magnetic field, since it includes all the contributions from the external field (but is free from the point of view of QCD interactions). The second term, i.e., the normal ordered product, will be expanded in terms of the QCD condensates.

Our approach is analogous to the one used to obtain the quark propagator in the QCDSR method in nuclear matter (see, for example, Ref. [42] for a review). The main idea is that one can expand the matrix element $\left\langle: q_{a \alpha}(x) \bar{q}_{b \beta}(0):\right\rangle \equiv$ $\left\langle\Omega\left|: q_{a \alpha}(x) \bar{q}_{b \beta}(0):\right| \Omega\right\rangle$ in terms of the usual basis for the Dirac matrices, $\left\{1, \gamma_{\mu}, \gamma_{5}, \gamma_{5} \gamma_{\mu}, \sigma_{\mu \nu}\right\}$, where as usual $\sigma_{\mu \nu} \equiv i\left[\gamma_{\mu}, \gamma_{\nu}\right] / 2$. Thus, we see that

$$
\left\langle: q_{a \alpha}(x) \bar{q}_{b \beta}(0):\right\rangle=\delta_{a b}\left(a \delta_{\alpha \beta}+b_{\mu \nu} \sigma_{\alpha \beta}^{\mu \nu}\right),
$$

where $a, b_{\mu \nu}$ are determined below. In the vacuum, only the first term appears, by parity and time-reversal invariance. However, the external magnetic field breaks time-reversal invariance, and thus, the tensor term in Eq. (A2) is now allowed. Since the only tensor at our disposal is the external electromagnetic field $F_{\mu \nu}$, one sees that $b_{\mu \nu} \propto F_{\mu \nu}$. The quantities in Eq. (A2) can be obtained by suitable contractions of both sides with the appropriate Dirac matrices, which gives

$$
\begin{aligned}
\left\langle: q_{a \alpha}(x) \bar{q}_{b \beta}(0):\right\rangle= & -\frac{\delta_{a b}}{12}\left(\langle: \bar{q}(0) q(x):\rangle \delta_{\alpha \beta}\right. \\
& \left.+\frac{1}{2}\left\langle: \bar{q}(0) \sigma_{\mu \nu} q(x):\right\rangle \sigma_{\alpha \beta}^{\mu \nu}\right) .
\end{aligned}
$$

Since we are performing a short-distance expansion, we can Taylor-expand the quark field $q_{a}(x)$ for small $x$. In the fixedpoint gauge for the color gauge potential, $x^{\mu} A_{\mu}=0$, we have $x^{\mu} D_{\mu}=x^{\mu} \partial_{\mu}$. So, the Taylor expansion takes the form

$$
q_{a}(x)=q_{a}(0)+\left.x^{\mu} D_{\mu} q_{a}\right|_{x=0}+\left.\frac{1}{2} x^{\mu} x^{\nu} D_{\mu} D_{\nu} q_{a}\right|_{x=0}+\cdots .
$$

Since we are interested only in dimension-3 operators, we can truncate the Taylor expansion to the zeroth-order term, since keeping higher-order terms in the expansion corresponds to considering condensates of higher dimensions. With this expansion, we can write the nonperturbative part of the quark propagator (up to condensates of dimension 3) as

$$
\left\langle: q_{a \alpha}(x) \bar{q}_{b \beta}(0):\right\rangle \approx-\frac{\delta_{a b}}{12}\langle: \bar{q} q:\rangle \delta_{\alpha \beta}-\frac{\delta_{a b}}{24}\left\langle: \bar{q} \sigma_{\mu \nu} q:\right\rangle \sigma_{\alpha \beta}^{\mu \nu} .
$$

In our case, the magnetic field is in the $z$ (3) spatial direction. In this situation, the only nonzero magnetic condensates are $\left\langle: \bar{q} \sigma_{12} q:\right\rangle$ and $\left\langle: \bar{q} \sigma_{21} q:\right\rangle=-\left\langle: \bar{q} \sigma_{12} q:\right\rangle$. Thus, in this case,

$$
\left\langle: q_{a \alpha}(x) \bar{q}_{b \beta}(0):\right\rangle \approx-\frac{\delta_{a b}}{12}\langle: \bar{q} q:\rangle \delta_{\alpha \beta}-\frac{\delta_{a b}}{12}\left\langle: \bar{q} \sigma_{12} q:\right\rangle \sigma_{\alpha \beta}^{12} .
$$

\section{APPENDIX B: CORRELATOR IN THE WEAK FIELD LIMIT}

The diagrams with nonvanishing traces are shown in Fig. 8. In this notation, the line with the square corresponds to the term $\left(e B / m^{2}\right)$ or $\left(e B / m^{2}\right)^{2}$ of the weak field propagator expansion in Eq. (22).

The first diagram in Fig. 8 leads to 


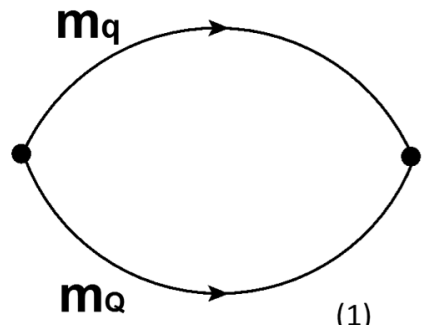

(1)

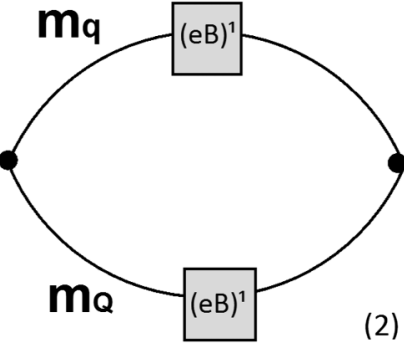

(2)

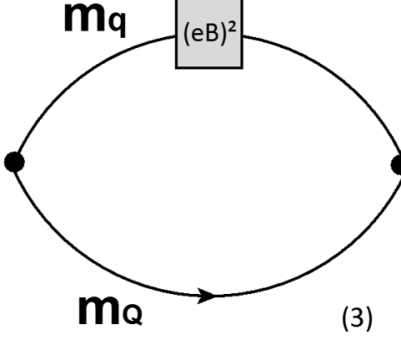

(3)

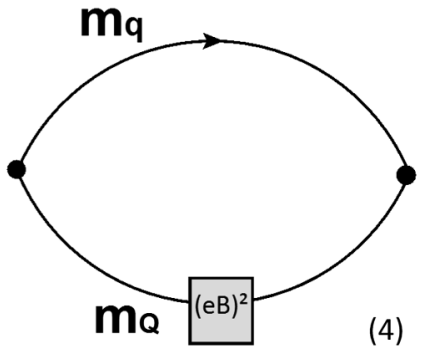

FIG. 8. Perturbative diagrams up to order $(e B)^{2}$.

$\Pi_{(1)}=3 \cdot 4 i \int \frac{d^{4} k}{(2 \pi)^{4}} \frac{k^{2}+k \cdot q-m_{q} m_{Q}}{\left(k^{2}-m_{Q}^{2}\right)\left((k+q)^{2}-m_{q}^{2}\right)}=3 \cdot 4 i \int_{0}^{1} d x \int \frac{d^{4} k}{(2 \pi)^{4}}\left[\frac{k^{2}}{\left(k^{2}-\Delta\right)^{2}}+\frac{\left(-x q^{2}(1-x)-m_{q} m_{Q}\right)}{\left(k^{2}-\Delta\right)^{2}}\right]$,

with $\Delta \equiv-x(1-x) q^{2}+x m_{Q}^{2}+(1-x) m_{q}^{2}$, where $x$ is a Feynman parameter. After integrating over the momentum, we obtain

$\Pi_{(1)}=\frac{3 \cdot 4}{(4 \pi)^{2}} \int_{0}^{1} d x \ln \Delta\left(2 \Delta-x q^{2}(1-x)-m_{q} m_{Q}\right)$.

This is the usual perturbative, $e B=0$, contribution to the correlator.

The second diagram in Fig. 8 corresponds to

$$
\begin{aligned}
\Pi_{(2)}= & 3 \cdot 4 i(c B)(C B) \int \frac{d^{2} k_{\perp}}{(2 \pi)^{2}} \int \frac{d^{2} k_{\|}}{(2 \pi)^{2}} \\
& \times \frac{k_{\|}^{2}+k_{\|} \cdot q_{\|}+m_{q} m_{Q}}{\left((k+q)^{2}-m_{q}^{2}\right)^{2}\left(k^{2}-m_{Q}^{2}\right)},
\end{aligned}
$$

with $k_{\|}=\left(k_{0}, k_{3}\right)$ and $k_{\perp}=\left(k_{1}, k_{2}\right)$. After the Feynman parametrization, we arrive at

$$
\begin{aligned}
\Pi_{(2)}= & 3 \cdot 4 i(c B)(C B) 6 \int_{0}^{1} d x x(1-x) \\
& \times \int \frac{d^{2} k_{\perp}}{(2 \pi)^{2}} \int \frac{d^{2} k_{\|}}{(2 \pi)^{2}} \\
& \times\left[\frac{k_{\|}^{2}}{\left(k_{\|}^{2}-\Delta_{\|}\right)^{4}}+\frac{\left(-x q_{\|}^{2}+x^{2} q_{\|}^{2}-m_{q} m_{Q}\right)}{\left(k_{\|}^{2}-\Delta_{\|}\right)^{4}}\right],
\end{aligned}
$$

with $\quad \Delta_{\|}=k_{\perp}^{2}-x(1-x) q_{\|}^{2}-x(1-x) q_{\perp}^{2}-x\left(-m_{q}^{2}+m_{Q}^{2}\right)+$ $m_{Q}^{2}$. Then, we can evaluate the $k_{\|}$integral. The same procedure applies to the $k_{\perp}$ integral. In the end, we obtain the following expression:

$$
\Pi_{(2)}=3 \cdot 4(c B)(C B) \frac{1}{(4 \pi)^{2}} \int_{0}^{1} d x x(1-x)\left[\frac{1}{\Delta_{\perp}}-\frac{\left(q_{\|}^{2}\left(x^{2}-x\right)-m_{q} m_{Q}\right)}{2 \Delta_{\perp}^{2}}\right],
$$

with $\Delta_{\perp}=\left(x^{2}-x\right) q^{2}+x m_{q}^{2}+(1-x) m_{Q}^{2}$.

The third diagram in Fig. 8 is given by

$$
\Pi_{(3)}=3 \cdot 4 i(c B)^{2} \int \frac{d^{4} k}{(2 \pi)^{4}}\left[2 \frac{\left(k_{1}^{2}+k_{2}^{2}\right)\left(m_{q} m_{Q}-k \cdot(k+q)\right)}{\left((k+q)^{2}-m_{q}^{2}\right)\left(k^{2}-m_{Q}^{2}\right)^{4}}-2 \frac{\left(k_{1} \cdot\left(k_{1}+q_{1}\right)+k_{2} \cdot\left(k_{2}+q_{2}\right)\right)}{\left((k+q)^{2}-m_{q}^{2}\right)\left(k^{2}-m_{Q}^{2}\right)^{3}}\right] .
$$

By the same procedure worked out for the second diagram, we obtain

$$
\begin{aligned}
\Pi_{(3)}= & \frac{3 \cdot 4}{(4 \pi)^{2}}(c B)^{2} \int_{0}^{1} d x(1-x)^{3}\left[\frac{1}{\Delta_{\perp}}+\frac{q^{2}\left(x-x^{2}\right)-q_{\perp}^{2}\left(x-4 x^{2}\right)+m_{q} m_{Q}}{3 \Delta_{\perp}^{2}}\right. \\
& \left.+\frac{2 x^{2} q_{\perp}^{2}\left(q^{2}\left(x-x^{2}\right)+m_{q} m_{Q}\right)}{3 \Delta_{\perp}^{3}}\right]-\frac{3 \cdot 4}{(4 \pi)^{2}}(c B)^{2} \int_{0}^{1}(1-x)^{2}\left[\frac{1}{\Delta_{\perp}}+\frac{q_{\perp}^{2}\left(x^{2}-x\right)}{\Delta_{\perp}^{2}}\right],
\end{aligned}
$$

with $\Delta_{\perp}=\left(x^{2}-x\right) q^{2}+x m_{q}^{2}+(1-x) m_{Q}^{2}$. In order to obtain the result for the fourth diagram, we can just replace $C \leftrightarrow c$, $m_{q} \leftrightarrow m_{Q}$, and $(q+k) \leftrightarrow k$ in the previous result for $\Pi_{(3)}$. 
[1] R. C. Duncan and C. Thompson, Astrophys. J. 392, L9 (1992).

[2] D. E. Kharzeev, L. D. McLerran, and H. J. Warringa, Nucl. Phys. A803, 227 (2008).

[3] V. Skokov, A. Y. Illarionov, and V. Toneev, Int. J. Mod. Phys. A 24, 5925 (2009).

[4] For an extensive list of references, see D. Kharzeev, K. Landsteiner, A. Schmitt, and H.-U. Yee, Strongly Interacting Matter in Magnetic Fields, Lecture Notes in Physics Vol. 871 (Springer, New York, 2013).

[5] G. S. Bali, F. Bruckmann, G. Endrodi, Z. Fodor, S. D. Katz, S. Krieg, A. Schafer, and K. K. Szabo, J. High Energy Phys. 02 (2012) 044.

[6] D. B. Lichtenberg, Int. J. Mod. Phys. A 02, 1669 (1987), and references therein.

[7] C. T.H. Davies et al. (HPQCD, UKQCD, MILC, and Fermilab Lattice Collaborations), Phys. Rev. Lett. 92, 022001 (2004).

[8] Z. Fodor and C. Hoelbling, Rev. Mod. Phys. 84, 449 (2012).

[9] M. A. Shifman, A. I. Vainshtein, and V. I. Zakharov, Nucl. Phys. B147, 519 (1979); B147, 385 (1979); B147, 448 (1979); T. Schafer, E. V. Shuryak, Rev. Mod. Phys. 70, 323 (1998); L. J. Reinders, H. Rubinstein, and S. Yazaki, Phys. Rep. 127, 1 (1985).

[10] For a review and references to original works, see, e.g., S. Narison, QCD as a Theory of Hadrons: From Partons to Confinement Cambridge Monographs on Particle Physics, Nuclear Physics and Cosmology (Cambridge University Press, Cambridge, 2007), 1 ed., Vol. 17, pp. 1-812; Qcd Spectral Sum Rules World Scientific Lecture Notes in Physics (World Scientific, Singapore, 1990), Vol. 26, pp. 1-527.

[11] B. L. Ioffe and A. V. Smilga, Nucl. Phys. B232, 109 (1984).

[12] B. L. Ioffe and A. V. Smilga, JETP Lett. 37, 298 (1983); 38, 48(E) (1983).

[13] J. Schwinger, Phys. Rev. 82, 664 (1951).

[14] I. Shushpanov and A. V. Smilga, Phys. Lett. B 402, 351 (1997).

[15] N. O. Agasian and I. Shushpanov, Phys. Lett. B 472, 143 (2000); N. O. Agasian, Phys. At. Nucl. 64, 554 (2001).

[16] T. D. Cohen, D. A. McGady, and E. S. Werbos, Phys. Rev. C 76, 055201 (2007); J. O. Andersen, Phys. Rev. D 86, 025020 (2012); arXiv:1205.6978.

[17] R. Gatto and M. Ruggieri, Phys. Rev. D 83, 034016 (2011).

[18] V. P. Gusynin, V. A. Miransky, and I. A. Shovkovy, Nucl. Phys. B462, 249 (1996).
[19] G. S. Bali, F. Bruckmann, G. Endrodi, Z. Fodor, S. D. Katz, and A. Schafer, Phys. Rev. D 86, 071502 (2012).

[20] P. Buividovich, M. Chernodub, E. Luschevskaya, and M. Polikarpov, Phys. Lett. B 682, 484 (2010).

[21] V. Braguta, P. Buividovich, T. Kalaydzhyan, S. Kusnetsov, and M. Polikarpov, Proc. Sci., Lattice2010 (2010) 190.

[22] M. D'Elia, S. Mukherjiee, and F. Sanlippo, Phys. Rev. D 82, 051501 (2010).

[23] M. D'Elia and F. Negro, Phys. Rev. D 83, 114028 (2011); M. D’Elia, Lect. Notes Phys. 871, 181 (2013).

[24] E.-M. Ilgenfritz, M. Kalinowski, M. Müller-Preussker, B. Petersson, and A. Schreiber, Phys. Rev. D 85, 114504 (2012).

[25] Y. A. Simonov, B. O. Kerbikov, and M. A. Andreichikov, arXiv:1210.0227.

[26] C. S. Machado, F. S. Navarra, E. G. de Oliveira, J. Noronha, and M. Strickland, Phys. Rev. D 88, 034009 (2013).

[27] K. G. Wilson, Phys. Rev. 179, 1499 (1969).

[28] L. J. Reinders, S. Yazaki, and H. R. Rubinstein, Phys. Lett. 104B, 305 (1981).

[29] E. V. Shuryak, Nucl. Phys. B198, 83 (1982).

[30] S. Narison, Phys. Lett. B 198, 104 (1987).

[31] R. D. Matheus, S. Narison, M. Nielsen, and J.-M. Richard, Phys. Rev. D 75, 014005 (2007).

[32] S. Narison, Phys. Lett. B 466, 345 (1999); 361, 121 (1995); 387162 (1996); 624, 223 (2005).

[33] S. C. Generalis and D. J. Broadhurst, Phys. Lett. 139B, 85 (1984).

[34] G. S. Bali, F. Bruckmann, M. Constantinou, M. Costa, G. Endrodi, S. D. Katz, H. Panagopoulos, and A. Schafer, Phys. Rev. D 86, 094512 (2012).

[35] V. Voronyuk, V. D. Toneev, W. Cassing, E. L. Bratkovskaya, V. P. Konchakovski, and S. A. Voloshin, Phys. Rev. C 83, 054911 (2011).

[36] K. Tuchin, Adv. High Energy Phys. 2013, 1 (2013).

[37] C. S. Machado, S. I. Finazzo, R. D. Matheus, and J. Noronha (to be published).

[38] M. Nielsen, F. S. Navarra, and S. H. Lee, Phys. Rep. 497, 41 (2010).

[39] J. Beringer et al. (Particle Data Group Collaboration), Phys. Rev. D 86, 010001 (2012).

[40] Y. Hidaka and A. Yamamoto, Phys. Rev. D 87, 094502 (2013).

[41] E. V. Luschevskaya and O. V. Larina, arXiv:1203.5699.

[42] T. D. Cohen, R. J. Furnstahl, D. K. Griegel, and X.-m. Jin, Prog. Part. Nucl. Phys. 35, 221 (1995). 